\title{
Overview of Palliative Care
}

\author{
Paudel BD ${ }^{1}$, Dangal G' ${ }^{2}$, Munday $\mathrm{D}^{3}$ \\ ${ }^{1}$ Department of Clinical Oncology, National Academy of Medical Sciences, Bir Hospital, Nepal, ${ }^{2}$ Department of Obstet- \\ rics and Gynaecology, Kathmandu Model Hospital, Nepal, ${ }^{3}$ Division of Health Sciences, Warwick Medical School, UK
}

Received: July 05, 2014; Accepted: October 12, 2014

Patients diagnosed with a life threatening condition like cancer suffer physically, socially and mentally. Their quality of life can be improved by effective communication and good symptom control even when the disease is incurable. Pain is the most common symptom and can be controlled by optimal use of analgesics especially oral morphine if severe. Psychosocial issues like depression and anxiety can be minimized by counseling and use of medicines. Death is more painful than birth but can be minimized by understanding the reality and by honest sharing which will help to minimize unnecessary sufferings. Palliative care is the care of these patients and their family members to make their life comfortable. Modern palliative care has developed around the world since the 1960s and in Nepal, since the beginning of 21st century. Much remains to be done before palliative care can be an integrated part of health care in Nepal. In this context gynaecologist having knowledge and skills in palliative care, have an important role in improving the quality of life of patients with life threatening conditions and their family members.

Keywords: gynecologist; Nepal; palliative care.

\section{INTRODUCTION}

The incidence of cancer, acquired immune deficiency syndrome (AIDS) and life threatening noncommunicable diseases, such as heart failure and chronic obstructive pulmonary disease (COPD) are increasing worldwide including in Nepal. ${ }^{1}$ Improved life expectancy, better diagnostic facilities and change of life style are all contributing to this shift. Each and every doctor including gynecologists will see patients with life-limiting chronic illness in their practice. Basic symptom control using a holistic approach is affordable and does not require highly specialized personnel, however this is often lacking. Lack of a basic understanding of palliative care amongst healthcare workers at all levels, a lack of training and confidence in communication skills and lack of knowledge of symptom control techniques hinders the delivery of optimal palliative care.

Palliative care worldwide is rapidly developing specialty. All doctors, including gynaecologists should be familiar with the basic principles of palliative care. Palliative care is an approach that

\section{CORRESPONDENCE}

Dr Bishnu Dutta Paudel

Department of Clinical Oncology, National Academy of Medical

Sciences, Bir Hospital, Nepal

Email: bisnupaudel@hotmail.com

Phone: +977-9841429570

improves the quality of life of patients and their families facing the problems associated with lifethreatening illness, through the prevention and relief of suffering by means of early identification and impeccable assessment and treatment of pain and other problems: physical, psychosocial and spiritual. ${ }^{2}$

\section{History}

Care of the sick has been a constant concern in society throughout history, the development of care of the dying in modern medicine is generally attributed to the work of the physician, Dame Cicely Saunders, who began her work with the terminally ill in 1948 and went on to establish the first modern hospice, St Christopher's Hospice, in London in $1967 .^{3}$ The term palliative care was introduced in Canada in 1976 marking the extension of hospice principles into hospital and community settings. The worldwide spread of palliative care in high and low income countries has been remarkable, with the first hospitalbased palliative care programs in the USA beginning in the 1980s and in India, palliative care developing in various places since the mid 1980s. Kerala has the most developed system of palliative care in India and was the first state to establish a palliative care policy in April 2008. ${ }^{4}$ 
In Nepal, we have an ancient tradition of care and attention for those who are old, ailing or dying. Establishment of a terminal care centre on the premises of Pashupati Nath Temple was an important step in the development of modern care of the dying in the country. Hospital based palliative care started with the establishment of oncology unit in Bir Hospital in 1991. In April 1999, Maiti Nepal established a hospice in Jhapa District, to shelter survivors of trafficking who were suffering from various illnesses, including hepatitis, tuberculosis and AIDS. Hospice Nepal, which started in 2000 with four beds in a Kathmandu hospital, now has its own building. Palliative care is provided in palliative care units at Bhaktapur Cancer Hospital (2004), Schechen Hospice and Clinic in Boudha (2004) and BP Koirala Memorial Cancer Hospital Bharatpur (2005). Thankot Hospice, offering free care, was opened in 2007 with the help of Korean friends, which is free. Nepal Network for Cancer Treatment and Research (NNCTR) has been involved in creating awareness, organizing workshops, seminars and training healthcare providers since 2002. Family Health International, Maiti Nepal and Nava Kiran Plus have also been involved in developing palliative and supportive care. ${ }^{5}$ Family Health International helps AIDS patients. Nava Kiran Plus is running care homes for people living with HIV. Nepalese Association of Palliative Care (NAPCare) was formed with its own constitution and was officially registered in 2009.

Worldwide Hospice Palliative Care Alliance categorised countries into four groups according to development of palliative care. ${ }^{6}$

Group 1 countries: where there is no known hospice and palliative care activity, including Afghanistan and Bhutan.

Group 2 countries: where there is evidence of initiatives to designed and create organisational, workforce and policy capacity for hospice-palliative care services to develop, but where no service has yet been established e.g. Qatar and Oman.

Group 3 countries: with localised hospice and palliative care provision e.g. Bangladesh, Pakistan, India and Nepal.

Group 4 countries: where palliative care is approaching integration into mainstream health services, e.g. most western European countries, North America, Australia, Japan, Singapore and Uganda.

\section{Models of Palliative Care}

The International Association of Hospice and Palliative Care (IAHPC) believes that all low income countries should be encouraged and enabled to develop their own appropriate models of palliative care, based on the needs of the patients and availability of resources and building on their established health systems, rather than follow models more appropriate to high income countries. ${ }^{7}$

Palliative care service operates in one or more of the following ways.

In-patient beds: These can be either in a palliative care unit, which is part of a hospital, or in an independent freestanding unit. For instance hospital units may be in a special ward within the hospital or in a separate unit in the hospital grounds. Freestanding units are separate from the main hospital, but should be able to access hospital clinical services. Inpatient units are useful for complex symptom management (physical or psychosocial), respite and end of life care.

Community services: Many models of community based palliative care exist, with a team of clinicians managing the patient at home and providing advice and support for the family.

Day care units: Such units are often linked to inpatient palliative care units in hospitals or hospices or may be free standing. They provide symptom control, rehabilitation, and support for patients and families and respite for people cared for at home. Patients need to be well enough to travel to and from the unit.

Hospital palliative care teams: These are staffed by doctors and nurses who have received specialist palliative care training. They provide advice for patients and family members referred to them from all wards and specialties in the hospital and advise on all aspects of palliative care. An important part of their role is to provide support and education to hospital staff increasing their skills and understanding of palliative care and enabling them to provide high quality palliative care, preventing the need for transfer of some patients to specialist units. 


\section{Barriers to Delivering Palliative Care in Nepal}

Many patients with advanced disease do not receive optimal palliative care and others are referred too late in the course of their disease to benefit from treatment. The reasons for this may relate to different factors:

Lack of skilled manpower: Palliative care is a newly recognized specialty, so far we do not have a palliative care post-graduate training program in Nepal and there are no Nepali palliative care specialists.

Lack of knowledge and skills of palliative care among health workers: To address this problem some effort has been done. NNCTR sent some doctors and nurses to India for palliative care training and has conducted palliative care training in Nepal. Hospice Nepal has been conducting two day palliative care training courses for nurses and doctors; National Academy of Medical Sciences (NAMS) started palliative care training for its residents and fellows; BP Koirala Memorial Hospital and other institutions are also conducting palliative care training. National Health Training Center (NHTC) has adopted two weeks palliative care training for heath workers, which will be conducted annually.

Lack of understanding of palliative care by patients and relatives or the belief that palliative care is only for dying people. This is one reason why the facility in Pashupati Temple has not been developed and used by people optimally.

Barriers related to society and culture: Dying and death still remains, to a large extent, a taboo subject in Nepal. There is still a deeply held view that maintaining life - whatever its quality - is the prime responsibility of health care professionals and services and also the responsibility of family members.

Availability and accessibility of essential medicine especially oral morphine. Because of the joint effort of different organizations and professionals, morphine became available in Nepal in 2005. In 2008, because morphine supply was still irregular, working together with National Health Care Limited and other stakeholders an agreement was reached to start morphine production in Nepal. Finally in 2009 morphine production was started.
Lack of training and confidence of doctors in Nepal to use morphine to treat patients with severe pain.

Lack of a national palliative care policy: There are no designated beds or positions for health professionals in palliative care within government hospitals. However, in May 2014 the World Health Assembly in Geneva passed a palliative care resolution to which Nepal is a signatory, demonstrating the government's commitment to develop a palliative care program for Nepal.

\section{Role of Communication}

These days misunderstanding between doctors and patients are frequently reported in Nepal. Improved knowledge and skills in good communication will help to minimize this, enhancing day-to-day clinical practice at least for patients with advanced cancer.

A practical protocol that can be used by gynecologists to improve communication is the Context Listening Acknowledge Strategy Summary (CLASS) protocol. ${ }^{8}$ This five-step basic protocol for medical communication provides a framework for good communication in the clinical setting.

Context of the interview including the physical setting and conditions for good communication is important. Consulting in a private room if possible, arranging the space optimally, being at the same level as the patient to retain eye contact and avoiding having a desk between counselor and the patient all contribute to a good setting for the consultation. Next step is to introduce clinician to the patient. Using open body language - leaning forward slightly, maintaining 'soft' eye contact and using touch if appropriate is helpful. A few seconds spent establishing these features of the initial communication/interview and making the patient feel at ease will aid communication and save time by preventing frustration and misunderstanding later. Interruptions by doctor by responding to the telephone, pager, or a colleague should be avoided. This can be interpreted as an insult by the patient. If it is not possible to avoid calls or turn off your mobile phone, then explain to the patient that you are sorry about any interruption, explain the reason for example dealing with a sick patient and that you will resume the interview as soon as possible. 
Listening is vital in a good consultation. Patients and family members frequently complain that doctors do not listen to them. Let the patient speak without interruption. Start the interview with open questions rather than closed questions requiring 'yes' or 'no answers only; avoid leading questions. Facilitate the interview by encouraging the patient to talk with the use of gestures like nodding, smiling, saying 'yes,' 'hmm,' 'please go on,' 'tell me more' etc, maintaining eye contact.

Acknowledgment of emotions is an important way of showing empathy towards the patient and indicating that you value him/her as a person. This is a particularly important technique in emotionally charged conversations for example the patient becomes tearful or a relative becomes angry. If the patient feels sad, the doctor should acknowledge this and say that they can understand that the information shared is upsetting. If the relative is angry, acknowledge this by saying something such as "I can see that this has made you feel angry, can you tell me why?" If the patient is not displaying emotions, but you have shared bad news, ask them gently, but directly, 'how has this made you feel?' and help them to explore their feelings and warn them that their feelings may change and that this is normal.

Doctors are likely to have a preferred management plan or strategy. Check that the patient understands what you have told them about their condition; ask the patient if they have any questions and try to answer them in a clear and jargon-free way. Then explain clearly and simply your management plan, any alternatives which might be possible and ask the patient if they have further questions. Once management has been agreed, explain this clearly step by step, pausing again to check understanding and answer questions.

The Summary is the closure of the interview/ consultation. This is when you make sure that the patient and family have understood and do not have any further questions. Ending the interview has three main components namely; making a summary of the main topics you have discussed, checking the patient and family have understood the plan and asking them to repeat it back to you.

\section{Pain Management}

Pain is a common symptom, which a doctor has to address in clinical practice and palliative care, however patients often do not get adequate pain relief. Improvement in pain control can be achieved by following a few simple procedures as we set out in this section.

First we should understand that pain is real - pain is what the patient says hurts. The International Association for the Study of Pain (IASP) has defined pain as an unpleasant, subjective, sensory and emotional experience associated with actual or potential tissue damage or described in terms of such damage. ${ }^{9}$ Basically there are two types of pain nociceptive pain and neuropathic pain. Nociceptive Pain refers to pain resulting from activation of peripheral nerves through the stimulation of nociceptors. Neuropathic pain refers to pain arising from nerve damage in the peripheral or central nervous system.

Pain assessment is a very important component of pain management, enabling the clinician to diagnose the likely cause of pain and therefore to target analgesia appropriately. In order for pain assessment to be thorough the OPQRST mnemonic can be used.

O-stands for the onset of pain - when and how does it start?

P- provoking or palliating factors,

Q -quality of pain (sharp, dull, burning, colic etc),

$\mathrm{R}$ - does the pain radiate elsewhere,

$\mathrm{S}$-how severe is the pain,

T-time - how long does it last, is it continuous, does it come and go?

To assess the severity the Numerical Pain Scale (NRS) can be used. NRS scale ranges from 0 to 10. Patient are asked to say how severe the pain is, where 0 means no pain and 10 means the worst pain they can imagine. 1-3 is normally equated by physicians with mild pain, 4-6 - moderate pain and 7-10 - severe pain.

Every clinician should know the WHO analgesic ladder guidelines for the management of pain. By using this simple guideline more than $80 \%$ of patients with pain may be given effective analgesia. ${ }^{10}$ Mild pain is treated by non-opoids like paracetamol and/ 
or non-steroidal anti-inflammatory drug (NSAID) such as ibuprofen. The dose of paracetamol is $1 \mathrm{gm}$ four hourly which can be safely prescribed to all patients apart from those with severe liver disease when the dose should be reduced. If paracetamol is insufficient to control pain, mild opioids like codeine or tramadol can be prescribed. For severe pain, where mild opioids are not controlling the pain, morphine is the medicine of choice (Figure 1).

STEP 1: Non-opioid for mild pain (eg. paracetamol,
NSAIDs) + adjuvant
STEP 2: Opioid for moderate pain (eg. tramadol, codeine)
+non-opioid + adjuvant
STEP 3: Opioid for strong pain (eg. morphine) +non-
opioid + adjuvant
Figure 1. Severity of pain and its management as
per WHO analgesic ladder.

Morphine is an economical and safe medicine, which is available in 'immediate release' and 'slow release' preparations. It should be given regularly to ensure smooth analgesia. Usually $2.5 \mathrm{mg}-5 \mathrm{mg}$ four hourly is the starting dose (given as an 'immediate release' syrup (concentration $10 \mathrm{mg} / 5 \mathrm{ml}$ ). This dose can be slowly increased as needed until the patient gets adequate pain control with tolerable side effects. If a patient is receiving $10 \mathrm{mg}$ doses, a $10 \mathrm{mg}$ immediate release tablet can be used instead of morphine syrup. Once we know the effective pain-relieving dose using immediate release preparations these can be converted to twice daily slow release tablets (available $10 \mathrm{mg}$ and $30 \mathrm{mg}$ strengths). Patients on slow release morphine should also be given immediate release morphine in case they get an increase in pain in between doses (known as breakthrough pain). The dose of immediate release morphine for breakthrough pain is one sixth of the total daily dose. Morphine injections should only be used if a patient is unable to swallow morphine or is vomiting and unable to retain it. Oral morphine in palliative care is otherwise as effective as by injection.

Constipation is the most common side effect of morphine for which a laxative should be prescribed. Respiratory depression is very rare as long as morphine is titrated as described above. A patient is much more likely to become drowsy and sedated before developing respiratory depression. It is important to remember that patients may be suffering from psychological, social and spiritual issues and unless these are addressed it could be difficult to control their physical pain even when using morphine according to the guidelines.

Co-analgesics are medicines primarily used for other purpose but can be used to relieve pain. Neuropathic pain, which is typically burning or shooting in nature is often not very responsive to morphine and requires tricyclic antidepressants e.g.amitriptyline $10-75 \mathrm{mg}$ at night or anticonvulsants e.g. sodium valproate $100-1200 \mathrm{mg}$ daily in divided doses. Co-analgesics should be started at the lowest dose and should be increased slowly each 2-3 days until the pain is under control. Drowsiness is the commonest side effect for these drugs. Also only 1 in 3 patients given either drug will benefit from it.

\section{Bowel Obstruction and its Management}

Bowel obstruction is a common problem particularly in ovarian cancer. It is due to mechanical obstruction of the bowel lumen and/or peristaltic failure. We have to exclude faecal impaction by taking a comprehensive history and performing a rectal examination and abdominal X-ray. The clinical picture depends on the level, type and duration of the bowel obstruction. Clinical features of early obstruction are: constipation and intermittent nausea often relieved by vomiting undigested food. As the condition worsens symptoms include increased nausea and/ or faeculent vomiting (as small bowel contents are colonized by colonic bacteria), continuous abdominal pain due to tumour and/ or nerve infiltration (e.g. coeliac plexus involvement) and colic (in mechanical obstruction). Clinical signs may include altered bowel sounds and abdominal distension but these may be absent particularly in gastro-duodenal obstruction or in patients with extensive peritoneal spread. A nasogastric tube may be needed to control vomiting initially, but long-term use, which is uncomfortable for the patient, should be avoided.

Surgery for malignant bowel obstruction due to ovarian cancer is rarely indicated, as it will not normally be successful in relieving the obstruction. Contraindications to surgery are: diffuse intraabdominal cancer seen at previous surgery, diffuse, 
palpable intra-abdominal masses, massive ascites that recurs rapidly after drainage and high obstruction involving the proximal stomach.

\section{Medical management can include:}

Initially metoclopramide $10-20 \mathrm{mg}$ three times daily by injection as a prokinetic. This should be stopped if it leads to colic.

Hyoscine butylbromide 20-40 mg three times daily by injection can be used if metoclopramide is not successful or not tolerated. This will treat colic and also reduce intestinal secretions, which will lessen the amount of vomiting. Octreotide infusion 300$600 \mathrm{mcg} / 24$ hour subcutaneously can also reduce vomiting.

Fentanyl injection or patch for controlling stable, moderate to severe pain in patients with/ or at risk of peristaltic failure is less constipating than morphine and other opioids. ${ }^{11}$ Dexamethasone $(16 \mathrm{mg})$ for $4-7$ days may reverse partial obstruction. ${ }^{12}$

\section{Carcinoma of Cervix}

Women with life threatening conditions like cancer commonly face a wide range of complex and distressing physical and psychosocial symptoms including pain, ascites, bowel obstruction, dyspnoea, anxiety, fistula and ureteric obstruction. Such symptoms prove challenging to the clinicians who care for these women in a wide range of settings, from tertiary hospitals to rural and remote communities. In Nepal clinicians must provide psychological, physical, social and spiritual care to address multifactorial, often inter-related symptoms with little access to resources and support.

Carcinoma of cervix is the most common cancer in women in Nepal. Patients with advanced or recurrent cervical cancer may suffer from bleeding, fistulas and other symptoms. Vaginal bleeding may be controlled by vaginal packing, radiotherapy, embolization of the uterine arteries, surgical resection, and arterial ligation. Urinary fistulas although not necessarily painful, can have an extremely negative impact on quality of life. Patients with fistulas may often choose to avoid social and family encounters because of constant odour from fistula drainage. Palliation can be by creation of a ureterointestinal conduit, placement of bilateral percutaneous nephrostomies - preferable if life expectancy is less than a few months, however, tubes may become kinked, dislodged or become a source of infection. Alternatively, external pad (diapers), to absorb drainage, is also an option. Rectovaginal fistulas occur from primary tumor invasion of the adjacent rectum. A diverting colostomy is an option for control of discharge. ${ }^{13}$

\section{End-of-Life-Care}

Gynecologists spend years learning about birth. It may be useful to spend few minutes to reflect on death. Sometimes a terminally ill patient, with a few days to live, undergoes multiple investigations and is even admitted to the Intensive Care Unit (ICU) even though everybody knows it will not benefit the patient. Such a patient would have been much better managed with her husband, children and loved ones enabling them to say 'goodbye.'Instead the patient dies with little dignity, with uncomfortable IV and drainage tubes, surrounded by people she does not know. Such futile treatment can be a great financial burden to family members also.

Patients who are known to be dying should be treated with dignity and have their symptoms effectively controlled. Their doctors need to be open and honest with them, acknowledging to the patient and family that they are dying and answering their questions honestly and supporting them gently. Relatives are often concerned about the patient not eating and drinking. However, it is natural for dying patients not to take much by mouth and they should not be forced to do so. Intravenous fluid and NG tubes are also not needed. Patients do need good mouth care to treat thirst and prevent build up of coating on their tongue and mouth. Time needs to be spent in gently explaining these things to the family.

\section{Psychosocial Issues}

Psychosocial problems are common in terminally ill patients especially in women because of their very important position in family in a country like Nepal. It is important that these problems are identified and addressed in a holistic manner. 
Patients may display 'denial' where they are unable to accept or believe their diagnosis or prognosis. This is a normal defense mechanism and should not be forcefully broken. ${ }^{14}$ However, it is often possible to gently explore the patient's fears and expectations and to answer their questions honestly and gently and enable them to understand and acknowledge their condition. Relatives may try to protect the patient from the truth, believing that they are unable to cope with the reality of their situation. This can lead to lying to the patient and a barrier being erected between the patient and their family. The family may try to get the doctor to promise to not tell the patient about their diagnosis and prognosis. Considerable time and care needs to be taken in explaining to the relatives the dangers of not being honest with the patient. Promising to speak gently to the patient and not forcing unwanted information on them could assist in reassuring the family. Normally it is best to tell the patient and family about their illness together.

Anxiety and depression are common in patients with advanced cancer. Symptoms of depression are reported in $75 \%$ of patients with life-threatening illnesses. ${ }^{15}$ Taking a supportive approach to both patients and their families, exploring their fears and answering their questions truthfully and gently, can lessen anxiety and depression. Depression is more common if the patient is not told the truth about their condition and prognosis and they suspect that their condition is more serious than they have been told. When a supportive approach is not successful in treating depression antidepressants can be helpful. Anxiety prevalence is about $10 \%$ in palliative care. ${ }^{16}$ Anxiety should be addressed by exploring the patient's fears and giving them reassurance as far as you are able, without being dishonest. Sometimes just being able to express their fears is enough to alleviate anxiety. If a patient is worried about symptoms such as pain, you can reassure her of your intention to monitor and treat her pain very closely. If a patient has disabling anxiety e.g. panic attacks, then a small dose of lorazepam $0.5-1 \mathrm{mg}$ four times daily can be helpful, but they should still be supported psychologically.

\section{CONCLUSIONS}

Palliative care is a new specialty and specialists are not available in Nepal. Basic knowledge and skill in different aspects of palliative care from physical symptom management to psychosocial care by all will help minimize the suffering of patients diagnosed with advanced life threatening conditions and their family members.

\section{DISCLOSURE}

The authors report no conflicts of interest in this work.

No violation of human rights and safety.

Funding: Nil

\section{REFERENCES}

1. Pradhananga KK, Baral M, Shrestha BM. Multi-institution hospital-based cancer incidence data for Nepal: an initia report. Asian Pac J Cancer Prev. 2009;10(2):259-62.

2. WHO Definition of Palliative Care. [Cited 2014 October 12]. Available from http://www.who.int/ cancer / palliative definition/en/

3. Clark D. From margins to centre: a review of the history of palliative care in cancer. Lancet Oncol. 2007;8(5)430-8.

4. Paleri AK. Showing the way forward: pain and palliative care policy of the government of Kerala. Ind J Palliat Care. 2008;14(1):51-4.

5. Gautam P. Palliative care services in Nepal. KUMJ. 2006;4(16):405-8

6. Global Atlas of Palliative Care at the End of Life, 2014. [Cited 2014 October 12]. Available fromhttp://www.thewpca.org/ resources/global-atlas-of-palliative-care/
7. Doyle D, Woodruff R. The IAHPC manual of palliative care. 2nd ed. [Cited 2014 November 19]. Available from http:// www.hospicecare.com/iahpc-manual/IAHPC manual.htm

8. Buckman R. Communication skills in palliative care: apractical guide. Neurol Clin. 2001;19:989-94.

9. Rajagopal MR. Pain - basic considerations. Indian J Anesth. 2006;50(5):331-4.

10. Schug SA, Zech D, Dörr U. Cancer pain management according to WHO analgesic guidelines. J Pain Symptom Manag. 1990;5(1):27-32.

11. Mercadente S, Caligara M, Sapio M, Serreta R, Lodi F. Subcutaneous fentanyl infusion in a patient with bowel obstruction and renal failure. J Pain Symptom Manag. 1997;13(4):241-4. 
12. Thaker DA, Stafford BC, Gaffney LS. Palliative management of malignant bowel obstruction in terminally ill patient. Ind J Palliat Care. 2010;16(2):97-100.

13. Segreti EM, Levenback C, Morris M, Lucas KR, Gershenson DM, Burke TW. A comparison of end and loop colostomy for fecal diversion in gynecologic patients with colonic fistulas. Gynecol Oncol. 1996;60(1):49-53.

14. 1 Vos MS, De Haes JC. Denial in cancer patients: an explorative review. Psychooncology. 2007;16:12-25.
15. Axtell A. Depression in palliative care. J Palliat Med. 2008;11(3):529-30

16. Mitchell AJ, Chan M, Bhatti H, Halton M, Grassi L, Johansen $\mathrm{C}$, et al. Prevalence of depression, anxiety, and adjustment disorder in oncological, haematological, and palliative-care settings: a meta-analysis of 94 interview-based studies. Lancet Oncol. 2011;12:160-74. 